\title{
AN UNBALANCED MULTIPLE DESCRIPTION CODING SCHEME FOR VIDEO TRANSMISSION OVER WIRELESS AD HOC NETWORKS
}

\author{
Li Bin, Huang Feng, Sun Lifeng and Yang Shiqiang \\ Department of Computer Science \& Technology \\ Tsinghua University \\ Beijing 100084, P.R. China
}

\begin{abstract}
Video transmission over wireless ad hoc networks is hampered by packet losses. Even a single packet loss may cause error propagation until an intra-coded frame is received. Indeed, packet losses greatly degrade the video quality. In this paper, we propose an Unbalanced Multiple Description Coding (UMDC) scheme over a single path which requires only one single path as additional links are difficult to be guaranteed in reality and is capable of quickly recovering from packet losses and ensuring continuous playback. The proposed scheme uses two descriptions, the High-Resolution (HR) description and the Low-Resolution (LR) one. It uses the 'peg frames' to limit error propagation in the HR description. The two descriptions can help each other recover from packet losses. The simulation results show that the proposed UMDC scheme over a single path has a comparable performance with our UMDC scheme with multiple path transmission (MPT) and has a better viewing experience than the state-of-the-art FEC-based scheme.
\end{abstract}

\section{INTRODUCTION}

Video transmission over wireless ad hoc networks is hampered by packet losses. In fact video coders commonly use predictive coding scheme to reduce temporal correlation since it achieves better compression efficiency. And yet the main drawback of these schemes lies in even a single packet loss may cause errors that propagate in time during the decoding process. A general method to limit error propagation is to insert intra coding frames. However the high bit rate makes it particularly difficult to apply in many applications. Forward Error Correction (FEC) codes can also be deployed to increase the robustness of the coded bit-stream and thus reduce the length of error propagation [1]. Nevertheless, the obvious obstacle thrown in the way of FEC codes is that its correction capability is fixed when given a predetermined redundancy. A pattern of excessive packet losses essentially results in incapability to correct errors, thus causing severe quality degrada-

This work is supported by the National Natural Science Foundation of China under Grant No. 60503063. tion. Another well-known error-protection method, i.e. Automatic Repeat reQuest (ARQ) technique is not suitable for live video applications because of the stringent constraints on the packet delivery deadlines [2].

Fortunately, an alternative approach to tackle the problem of error propagation is applying Multiple Description Coding (MDC) techniques [3]. Attractive feature of low delay property makes MDC most promising especially for live video applications. MDC approach generally consists in generating two or more descriptions (MDC with two descriptions is fairly usual and herein it is illustrated as an example in the following), while source descriptions are packetized independently and then sent to the receiver over different paths. As long as these descriptions do not encounter packet losses concurrently, an acceptable quality could be obtained. In balanced MDC approaches [4] the two descriptions have the same importance while in unbalanced MDC [5] one description has a higher quality than the other. Since in UMDC, the Low Resolution (LR) description is primarily used as redundancy and exploited to conceal errors in the High Resolution (HR) description, UMDC gives a better control on the amount of introduced redundancy, compared with BMDC.

Previously we have committed many research efforts towards transmitting UMDC over multiple paths and observed encouraging results [6]. However, although exploiting path diversity indeed decreases the correlation among the descriptions [7][8], it complicates existing underlay network topology and requires additional link resource in reality. Particularly, for mobile ad hoc networks, what makes the multiple paths extremely difficult is the fact that there is no any guarantee on enough link resources. So UMDC over a single path is explored in [9]. Nevertheless, the simple LR-HR replacement policy adopted in [9] introduces the error-drift effect when any description encounters packet loss. Towards this end, we further improve our UMDC approach [6] to adapt to single path transmission (SPT).

Our scheme has the following advantages.

1. It can prevents error propagation in the HR description with the 'peg frames'.

2. It can recover quickly from packet losses with the mu- 
tual help of the HR and LR descriptions.

3. It requires only a single path to transmit video by using our packetization method.

The rest of this paper is organized as follows. We describe our proposed UMDC scheme in Section 2. And in Section 3, we present a performance comparison between the FEC and UMDC systems. Finally, we conclude in Section 4 with a summary of our work.

\section{OVERVIEW OF THE SCHEME}

\subsection{Our UMDC coders}

Our proposed UMDC coders are shown in Fig. 1 and Fig. 2. One standard encoder (H.264 in our experiments) produces the HR bit-stream and another produces the LR bit-stream. The bit-rate of the LR bit-stream is designed to be significantly lower than the one of the HR bit-stream. Note that the input of the LR encoder is the reconstructed frames of the HR encoder. The two standard encoders are unaltered for all the frames except for the 'peg frames'. We define the reconstructed $I$ frame (intra-coded) of the LR encoder as the 'peg frame'. The 'peg frame' is put into the frame buffer of the HR encoder instead of the current reconstructed $P$ frame of the HR encoder. And the next $P$ frame of the HR encoder is predicted using the 'peg frame' as the reference frame. The introduction of the 'peg frames' increases the robustness of the HR bit-stream against packet losses.

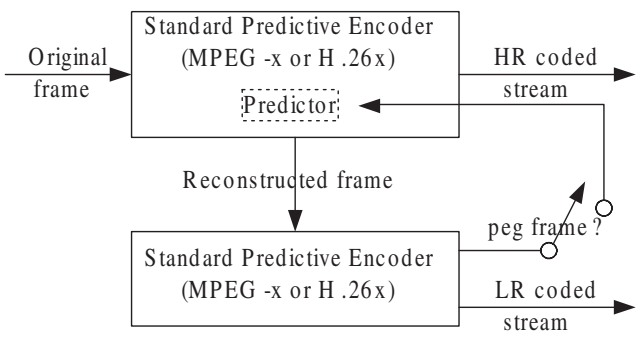

Fig. 1. Proposed UMDC video encoder.

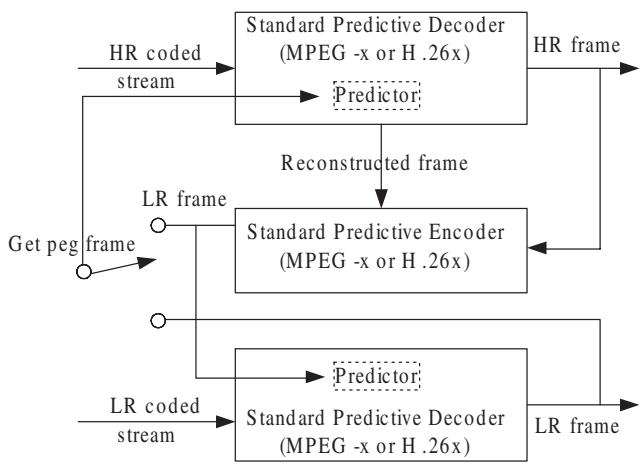

Fig. 2. Proposed UMDC video decoder.
At the UMDC decoder side, the HR decoder has to get the 'peg frame' for the motion compensation of the next $P$ frame. The 'peg frame' is available from the output of the LR decoder or from the reconstructed frames of a standard encoder. Note that the input of the standard encoder is the decoded data of the HR decoder. The LR decoder works as a standard decoder, except that sometimes it uses reconstructed frames of the standard encoder to keep the continuous decoding process.

The proposed UMDC coders are robust against packet losses. The examples are shown in Fig. 3 and Fig. 4 where $I_{p}$ denotes the 'peg frame', $P_{x}$ denotes the lost $P$ frame and $P_{d}$ denotes the discarded $P$ frame. When the HR and LR descriptions are both correctly received, both the two descriptions can be decoded correctly and the output is the HR data. When only the HR description encounters packet losses, in most of the common UMDCs, either the decoding process of the HR description pauses and the corresponding LR data is output or the corresponding LR data is used as the reference frames and the decoding process of the HR description continues. However, the two methods degrade the video quality because of the low quality of LR bit-stream or the mismatch of the HR encoder and the HR decoder. Therefore, we introduce the 'peg frames' to alleviate the problem. Because the next $P$ frame of the HR description uses the 'peg frame' as the reference frame, there is no mismatch between the HR encoder and decoder when we get the 'peg frame' from the correctly received LR description. The decoding process of the HR description restarts successfully, as illustrated in Fig. 3. When only the LR description encounters packet losses, the recovery process of our scheme is very simple. As mentioned in the above, we use the reconstructed video data from the HR encoder rather than the original video data, different from most of the common UMDCs. We can just simply input the reconstructed video data from the HR decoder into a standard encoder to generate the LR bit-stream. And the same LR bit-stream is produced and the decoding process of the LR description continues perfectly, as shown in Fig. 4. Compared to other UMDCs, our scheme avoid the drift when decoding the LR description.

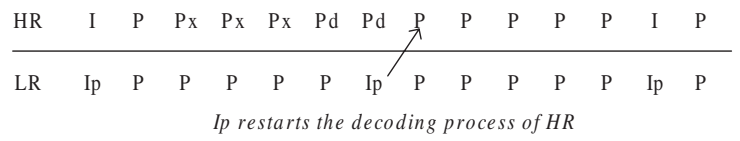

Fig. 3. A case of HR packet losses.

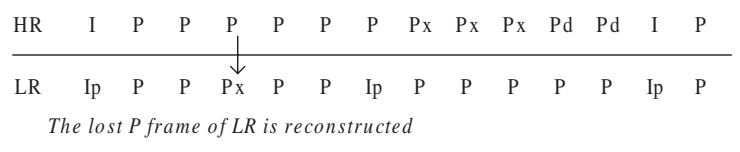

Fig. 4. A case of LR packet losses. 


\subsection{Our UMDC packetization method}

As mentioned in Section 1, our proposed UMDC scheme uses only one single path. Thus both the HR and LR descriptions need to be transmitted over the same path. In order to guarantee that the concurrence of packet loss for the two descriptions has a low probability, the corresponding HR and LR packets must not be transmitted simultaneously. Then we packetize the HR and LR data, which belongs to different frames respectively, into the same packet. Then the HR and LR data belonging to a same frame is transmitted over a path successively with an appropriate distance. The offset of the HR and LR data in the same packet reflects this distance. And it is determined according to the network conditions. When the network is in a good state, we use a smaller offset. Yet we use a larger offset when the network is in a bad state. The more small the offset is, the less low the introduced delay is. An example of our packetization method with the offset of 3 is illustrated in Fig. 5.

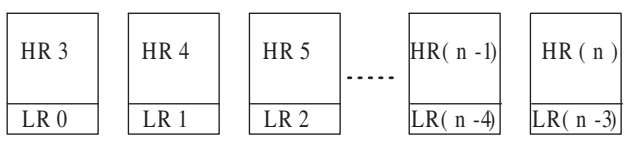

Fig. 5. An example of our packetization method.

\section{EXPERIMENTAL RESULTS}

This section presents the experimental results of the proposed UMDC scheme and the performance comparison with FEC. The comparison between the FEC system and the UMDC one is done at the same bit rate. That is, the HR bit rate equals the original one and the LR bit rate equals the bit-budget for FEC protection. For the FEC system, we implement a ReedSolomon code $(4,3)$. Since data interleaving is essential to guarantee good performance of the FEC codes with transmission affected by bursts, we also use a data interleaving that covers 4 frames. Fig. 6 shows the $(4,3)$ RS code employed for every frame and the packetization strategy every 4 frames.

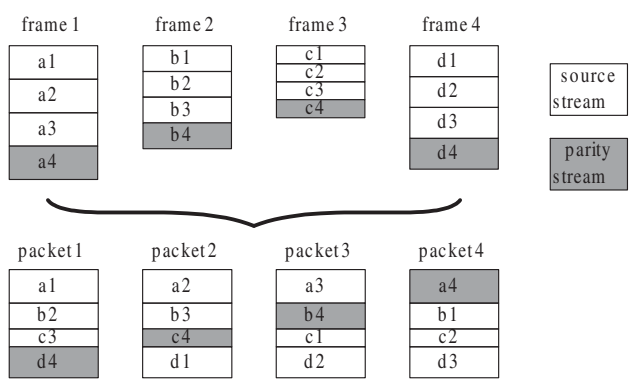

Fig. 6. (4, 3) RS code and packetization strategy for FEC system.
The Gilbert-Elliott model is used to model the bursty losses. When the state of the path is bad, all the packets are lost; when the state is good, the packets can still be lost due to collision or noise. The average dwell time in the good state is set to $1000 \mathrm{~ms}$. The average time in the bad state is varied from 20 to $300 \mathrm{~ms}$ to simulate the impact of channel burstiness.

We implemented the UMDC and FEC systems by modifying the JVT Software Version M8.6. We assume the RTP payload format for packetizing the H.264 video stream [10]. We test different video sequences and present the results of the Foreman sequence (QCIF, 15fps, 150 frames). Three metrics are used to evaluate the UMDC and FEC systems, including the average PSNR, the probability of pause and the PSNR variance. The average PSNR reflects the objective video quality while the two latter metrics illustrate the subjective video quality. The probability of pause represents the fluency of the playback and the PSNR variance represents the fluctuation of the video quality. We run the simulations over 50 different loss realizations with the same model parameters to get convictive results. The experimental results are illustrated in Fig. 7 - Fig. 9.

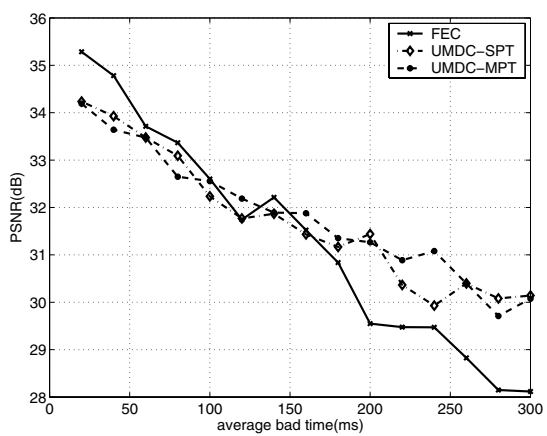

Fig. 7. PSNR versus average bad time of UMDC and FEC. Foreman QCIF sequence, HR (Original) bit rate $=100 \mathrm{kbps}$, LR (FEC protection) bit rate $=33.33 \mathrm{kbps}$.

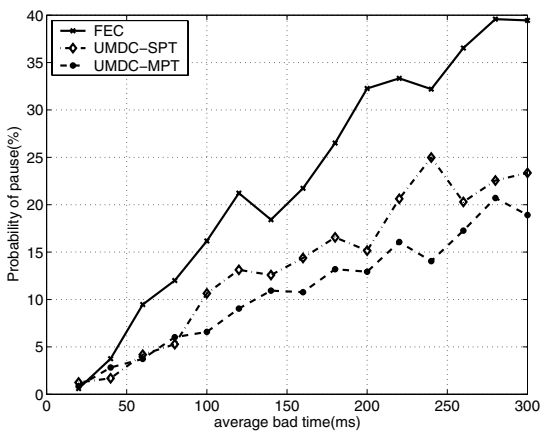

Fig. 8. Probability of pause versus average bad time of UMDC and FEC. IR period of $H R=50$ frames, IR period of $\mathrm{LR}=10$ frames, IR period of $\mathrm{FEC}=10$ frames. 


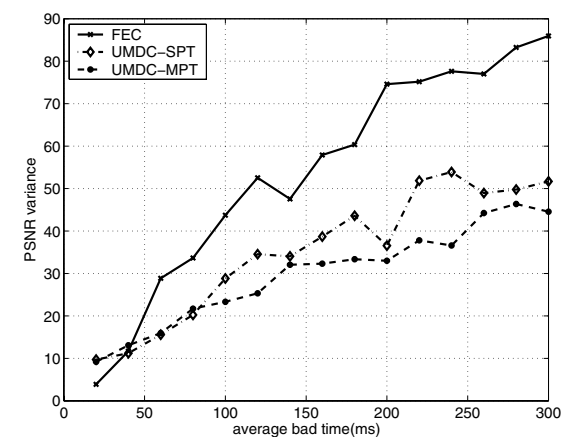

Fig. 9. PSNR variance versus average bad time of UMDC and FEC. Foreman QCIF sequence, HR (Original) bit rate $=100 \mathrm{kbps}$, LR (FEC protection) bit rate $=33.33 \mathrm{kbps}$.

Fig. 7 shows the decoded video quality of the UMDC with SPT or MPT and FEC systems for the Foreman sequence. The proposed UMDC with SPT scheme outperforms the FEC scheme when the average bad time is more than $180 \mathrm{~ms}$, in respect that: i) the FEC system effectively reconstructs the source data under the correction power of the $(4,3) \mathrm{RS}$ code; ii) in error-free environment, the PSNR of the HR stream is lower than the PSNR of the original stream at the same bit rate (100kbps). On the other hand, as the average bad time grows, the decoded quality of the FEC system degrades dramatically and the proposed UMDC with SPT system increases its performance gains over the FEC one.

In Fig. 8 we give the probability of pause against the average bad time. The probability of pause should be minimized since users are sensitive to the fluency of the video playback. As shown in Fig. 8, the proposed UMDC with SPT scheme achieves a lower probability of pause than the FEC scheme on almost all the test points. When the average bad time becomes large, the playback of the FEC-based stream is frequently interrupted, which bothers users greatly.

Besides the decoded quality and the probability of pause, we also adopt another metric, i.e. PSNR variance, to evaluate the UMDC and FEC systems. Fig. 9 shows that the proposed UMDC with SPT system has a smaller PSNR variance than the FEC one when the average bad time is over $20 \mathrm{~ms}$. This indicates that the proposed UMDC system is more stable than the FEC one when packet losses happen and gives users a better viewing experience.

In Fig.7 - Fig. 9, we can see that the performance of the UMDC with SPT and the one of the UMDC with MPT are comparable. This shows that our packetization method is effective to decrease the correlation of the HR and LR descriptions.

\section{CONCLUSION}

To achieve robust video transmission over wireless ad hoc networks, we propose an UMDC scheme with SPT capable of quickly recovering from losses and ensuring continuous playback. The simulation results under different conditions show that the proposed UMDC scheme with SPT has a comparable performance with our UMDC scheme with MPT and has a better viewing experience than the state-of-the-art FEC-based scheme.

\section{REFERENCES}

[1] L. Rizzo, "Effective erasure codes for reliable computer communication protocols," ACM Computer Communication Review, Apr. 1997.

[2] A. C. Begen, Y. Altunbasak, O. Ergun, and M. H. Ammar, "Multi-path selection for multiple description video streaming over overlay networks," Signal Processing: Image Communication, vol. 20, no. 1, pp. 3960, Jan. 2005.

[3] V. K. Goyal, "Multiple description coding: compression meets the network," IEEE Signal Processing Magazine, vol. 18, pp. 74-93, Sept. 2001.

[4] K. R. Matty and L. P. Kondi, "Balanced multiple description video coding using optimal partitioning of the dct coefficients," IEEE Transactions on CSVT, vol. 15, no. 7, pp. 928-934, Jul. 2005.

[5] M. Fumagalli, R. Lancini, and S. Tubaro, "A novel error-concealment algorithm for an unbalanced multiple description coding architecture," Proceedings of International Packet Video Workshop, Dec. 2004.

[6] Feng Huang, Lifeng Sun, and Yuzhuo Zhong, "A novel unbalanced multiple description coder for robust video transmission over ad hoc wireless networks," Proceedings of the 13th Multimedia Computing and Networking (MMCN), Jan. 2006.

[7] S. Ekmekci and T. Silora, "Unbalanced quantized multiple description video transmission using path diversity," Proceedings of SPIE's Electronic Imaging 2003, Jan. 2003.

[8] J. G. Apostolopoulos and S. J. Wee, "Unbalanced multiple description video communication using path diversity," Proceedings of International Conference on Imaging Processing, Oct. 2001.

[9] A. Vilei, G. Convertino, S. Oliva, and R. Cuppone, "A novel unbalanced multiple description scheme for video transmission over wlan," Proceedings of the 3rd ACM international workshop on Wireless mobile applications and services on WLAN hotspots, Sept. 2005.

[10] S. Wenger, T. Stockhammer, and M. M. Hannuksela, "Rtp payload format for h.264 video," Internet Draft, Mar. 2003. 